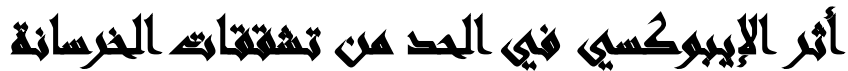

$[r]$

معن محمد صالح الرفاعي (')- يونس رجب محمد حسن علي (')

1) الهيئة العامة للتعليم التطبيقي والتدريب بدولة الكويت

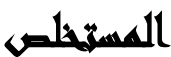

يهدف هذا البحث إلى التعرف على أنز مركب الإيبيوكسي في الحد من التشققات التي

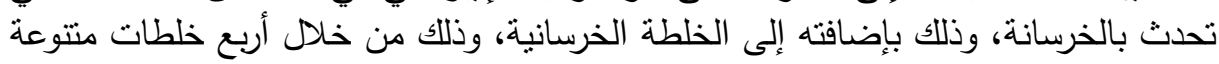

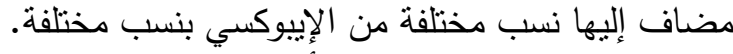

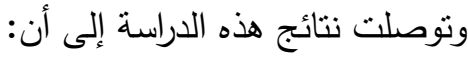

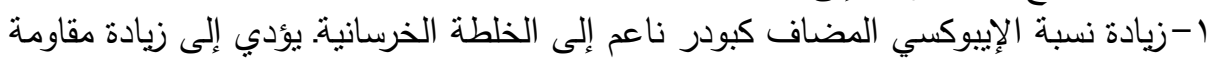

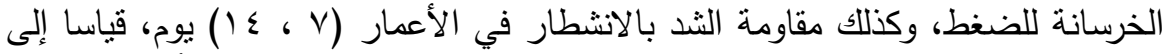

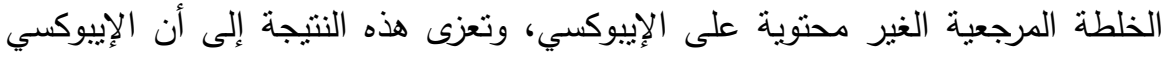

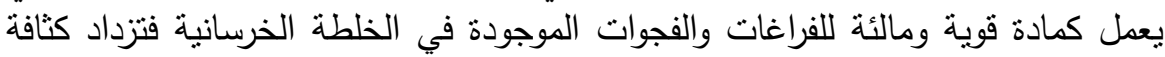

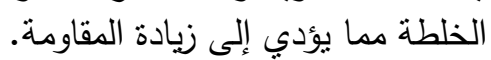

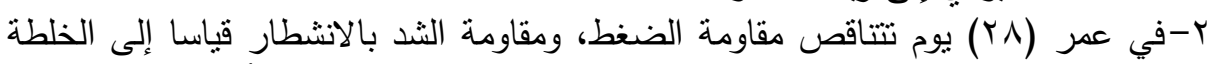

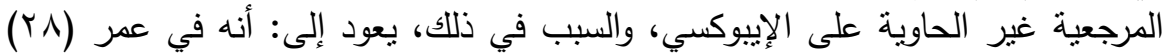

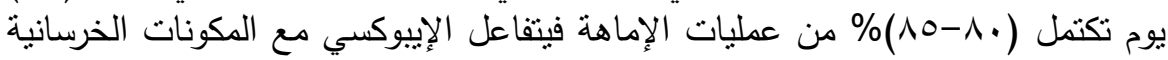

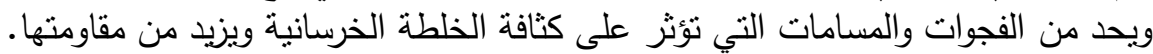

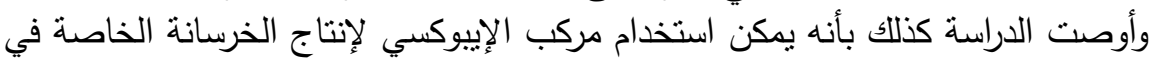

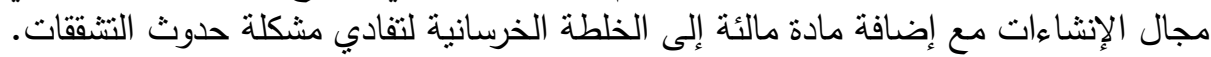
الكلمات المفتاحية: أثنر - الإيبوكسي - مقاومة الخرساتئة الخفانة للضغط - مقاومة الخرسانة للثند -

\section{Xax}

تعد الخرسانة المسلحة من المواد الأساسية التي تعتمد عليها العديد من البلدان حول

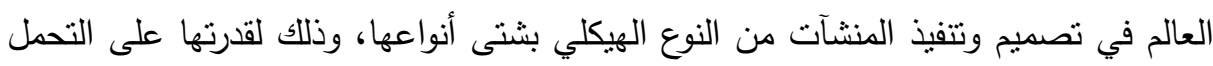

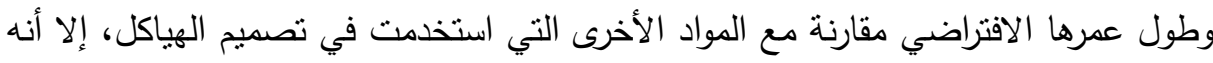
لا نزال هناك العديد من الإخفاقات الحادثة منل تآكل وصدأ حديد التسليح أو الإخفاقات في

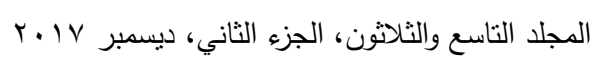


الخلطات الخرسانية والتي يؤدي حدوثها إلى تشقق بالعناصر الخرسانية المكونة للمبنى. (Jadhav, et al., 2016)

والخرسانة بطبيعتها مادة مسامية تحوي رطوبة، مما يُسهم في تسرب الماء والهواء لصلب التسليح، وبالتالي حدوث صدأ، ولقد أجرى العديد من الباحثين العمل التجريبي

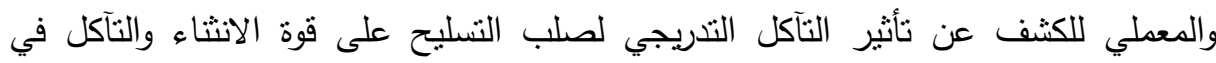
الحديد والذي يؤثر بدوره على العمر الافتراضي للهيكل الخرساني، بالطرق المختلفة، ومنها على سبيل المثال : طريقة التيار الكهربي. (Patil, 2011). وفي الآونة الأخيرة زاد اهتمام الباحثين بإدخال المركبات الكيميائية (الإضافات الخرسانية) في الخلطة بغرض تقليل التشققات في الخرسانة المسلحة ، وكذلك مقاومة الحرارة ، ومياه الأمطار ، والعوامل البيئية الأخرى التي تُسهم في التأثنر على خواص التيل الخرسانة، وتنساعد

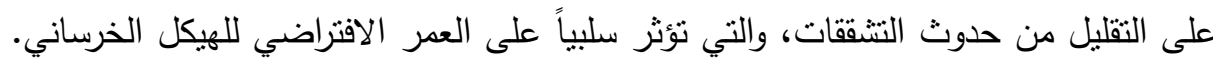
(Zhang, et al., 2017)

وتُعد مادة الإييوكسي من الإضافات الخرسانية التي استخدمت حديثاً وأثنتت فعاليتها بشكل منسارع، حيث إنه بدأ استخدامها في معالجة الأسطح الخرسانية من تشققات سبيتها

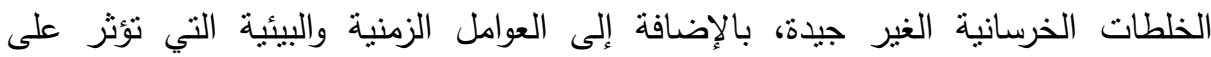

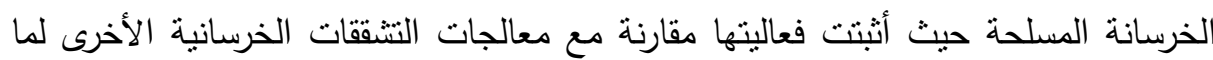

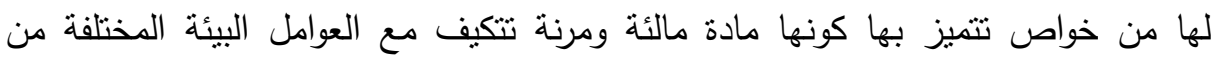

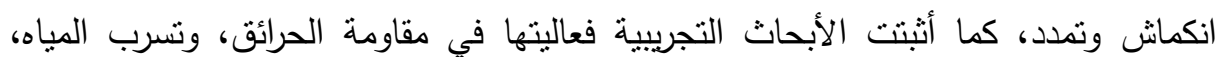
ومقاومة الرطوبة. (Gangarao, et al., 2007) ولما لها من خصائص ميكانيكية وفيزيائية جعلت منها مادة شديدة الالتصاق والصلابة التهابة في التعامل مع البيئات المختلفة، بالإضافة إلى انخفاض تكاليفها مقارنة مع الإضافات

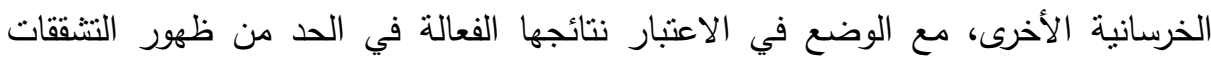
بالخرسانة المسلحة، وما يجعل منها مادة اقتصادية تطيل من عمر المبنى الخرساني، وتحد من ظهور المشكلات التي تظهر على الأسطح الخرسانية، والتي تؤثر على فعاليته. 
والإيبوكسي مادة قوية ومقاومة للتآكل، لما نوفره من مقاومة ممتازة للمواد الكيمبائية،

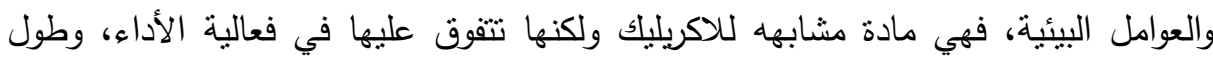

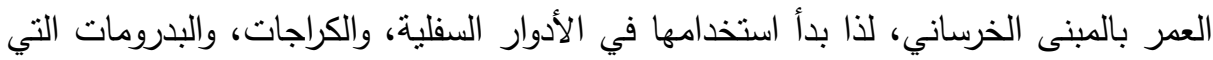
ترتفع فيها نسبة الرطوبة. (American society, 2008) وتطور استخدام الإيبوكسي والاعتماد عليه في علاج النتققات بالسدود والمباني الخرسانية الضخمة، لما لها من قدرة على علاج التشققات الخرسانية وعدم السماح بتسرب الماء والرطوبة إلى داخل الجسم الخرساني. (Scales, 1968) هذا بالإضافة إلى نتائج دراسة (Chung, 1975) حيث قام باختبار ثلاث قوالثم خرسانية للتحقق من كفاءة استخدام الإيبوكسي في الخلطة الخرسانية، والتي أكدت نتائجها على كفاءة القالب الخرساني بفضل إضافة الإيبوكسي في الخلطة الخرسانية، وصلابة القالب الباهي

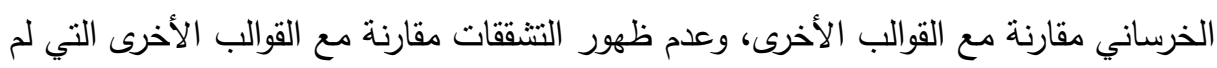
يستخدم فيها مادة الإيبوكسي. (Chung, 2016)

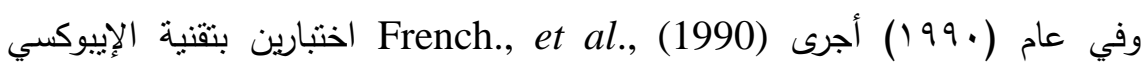

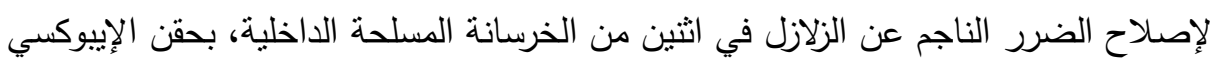
وأثثت الاختبارين أن مادة الإييوكسي عملت على القضاء على النتققات، واستعادة الخرسانة المتضررة من الزلازل القوة والصلابة مرة أخرى. (French, 1990) كما أكدت نتائج دراسة (2006) فعالية استخدام الإيبوكسي مع الخرسانة المسلحة حيث قام الباحثون على اختبار خواص مقاومة وتشوه العتبات الخرسانية المسلحة

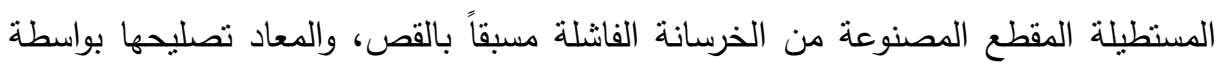
حقن التشققات بمادة الإيبوكسي، حيث تم استخدام خمس عتبات خرسانية مسلحة بسيطة

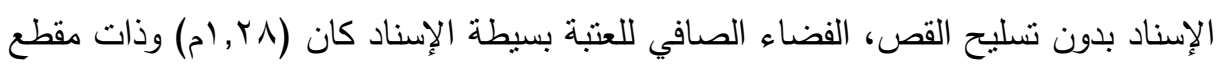

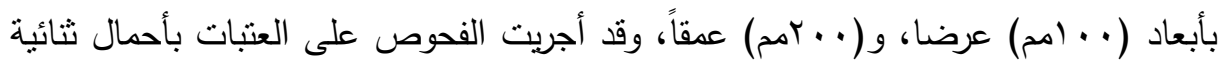

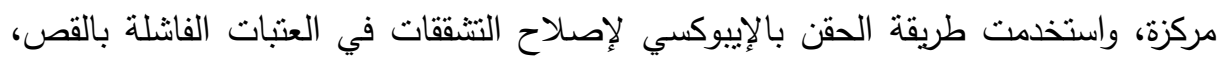

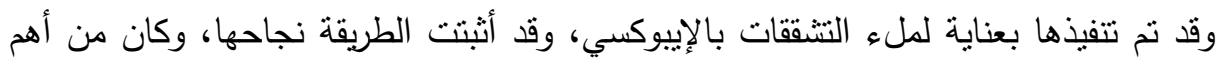

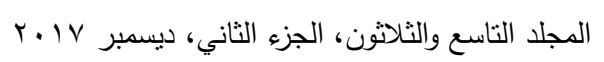


استتناجات التجربة هو نجاح طريقة الإصلاح في زيادة أو على الأقل استعادة مقاومة القص

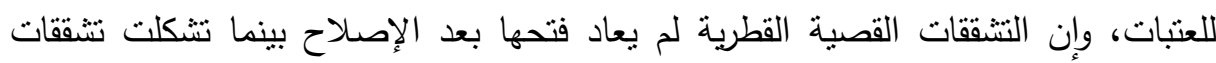
قصية قطرية جديدة قريبة، وبصورة عامة فإن السلوك الإنشائي للعنبات المصلحة كان مشابها لسلوك العتبات الأصلية مع جساءة أقل ومطيلية أعلى، وهو ما يُيرر فيله فعالية استخدام الإييوكسي مع الخلطات الخرسانية. (Bayan, 2006)

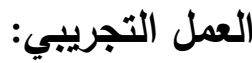

أولاً: المواد المستخدمة في التجربة: فيما يلي شرح موجز للمواد التي تم استخدامها في إجراء

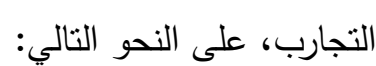
[1] الأسمنت: نم استخدام الأسمنت البورتلاندي الكويتي المقاوم للأملاح من إنتاج مصنع الكويت للأسمنت، والمطابق للمواصفات الكويتية.

[ץ] الركام الناعم: تم استخدام الركام الناعم (الرمل) من جنوب الجهراء بمحافظة الجهراء.

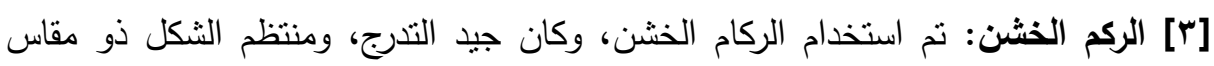
(• (ملم) وتم إيجاد التدرج الحبيبي له حسب المواصفات القياسية الكوينية. (Standard Specification for Concrete Aggregates) , [KWS GSO ASTM C33 / C33M:2015] [؛] المياه : نم استخدام المياه العادية من الثبكة الوطنية بدولة الكويت. [0] ايبوكسي قار: تم استخدام ايبوكسي قار في شكل البودر، وهو من المشتقات الكيماوية الحديثة التي تستخدم في مجالات كثيرة ومتعددة، والتي تم تطويعها في المجال المعماري لـاني

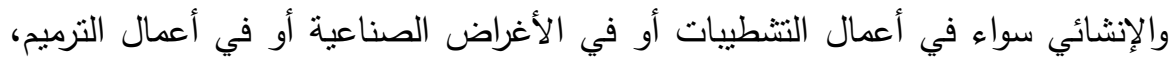
ويتكون الأييوكسي غالباً من مركبين أساسيين، وهما: أ - المركب الأول: الرزين Resin.

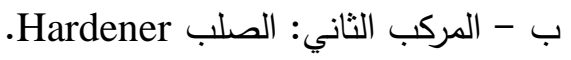


وكذللك يستخدم إييوكسي قار كمادة مالئة للفراغات والنتققات حيث يصلح لأرضيات الكباري ولمواسير الصرف الصحي، وهناك عدة احتياطات الواجب اتخاذها عند استعمال الايبوكسي لأنها مادة سامة ومضرة عند الاستتشاق، ومضره أيضاً على الجلد والعين لذلك فإنه يجب مراعاة الآتي عند استعمالها:

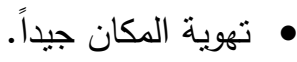
• • ارتداء القناعات والقفازات. • يجب الامتتاع عن التخخين أثناء التشغيل وتحضير وصب الخلطة.

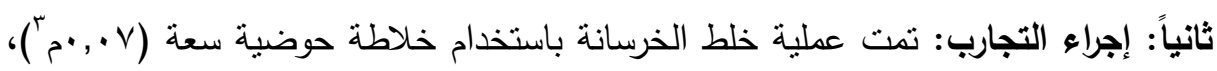
وكانت عملية الخلط تتم بوضع كمية من الأسمنت ثم كمية من الركام الناعم ثم كمية من الاييوكسي ثم كمية من الركام الخشن ثم تضاف الكمية المتنقية من الركام الخشن والناعم والأسمنت، والاييوكسي، ثم تتم عملية الخلط الجاف إلى أن تتم ملابسة الخلطة بالكامل ثم تثم

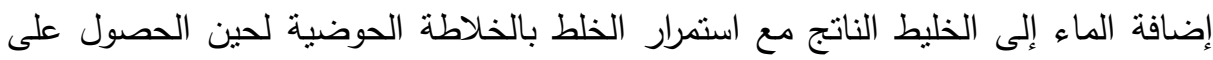
الخليط المتجانس، ومن ثم تتم عملية صب الخرسانة في القوالب على شكل طبقتين حيث تم

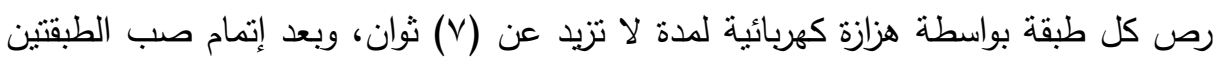
ورصها تمت معالجة سطح النماذج لغرض الحصول على سطح مستو .

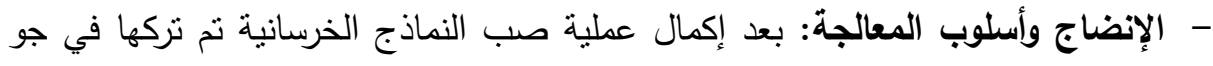
المختبر لمدة (ع Y) ساعة ثم فتح القوالب الحديدية وإخراج النماذج الخرسانية وتغطيسها

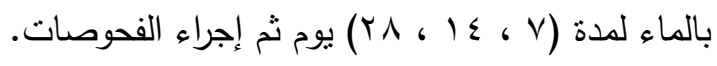
جدول(1): الخلطات الخرسانية المستخدمة

\begin{tabular}{|c|c|c|c|c|c|}
\hline \multirow{2}{*}{ 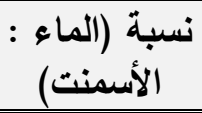 } & \multicolumn{4}{|c|}{ نسبة (الأسمنت، الرمل، الحصى، الأيبوكسي) } & \multirow{2}{*}{ سم الخلطة } \\
\hline & الايبوكسي & الحصى & 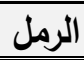 & الأسمنت & \\
\hline$\cdot, \mathrm{V}$ & V & $\Lambda, 0$ & $\Lambda, 0$ & 1 & A \\
\hline$\cdot, \mathrm{V}$ & 9 & $\Lambda, 0$ & $\Lambda, 0$ & 1 & B \\
\hline$\cdot, V$ & 14 & $\Lambda, 0$ & $\Lambda, 0$ & 1 & $\mathrm{C}$ \\
\hline$\cdot, \mathrm{V}$ & - & $\Lambda, 0$ & $\Lambda, 0$ & 1 & D \\
\hline
\end{tabular}


مجلة العلوم البيئية

معهد الدراسات والبحوث البيئية - جامعة عين شمس لهن 


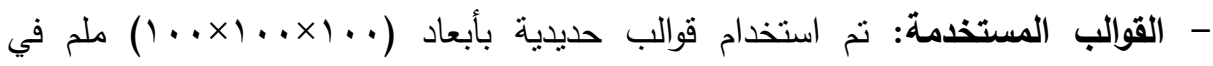

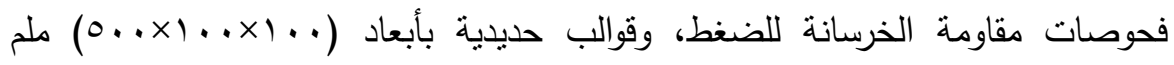

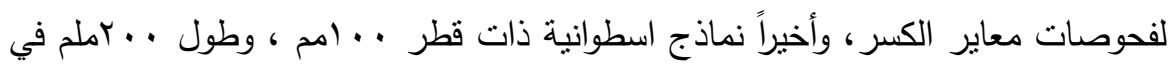
فحص الثد غير المباشر (الانفلاق). -

م فصص مقاومة الخرسانة للضفط: استعملت ماكنة الفحص من نوع (ELE) ذات سعة

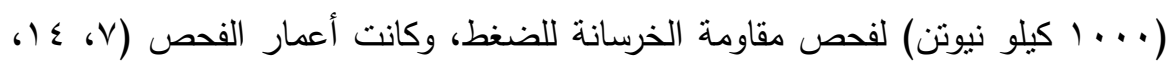

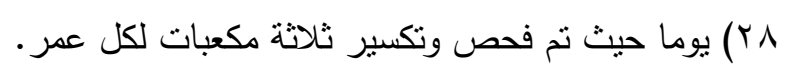

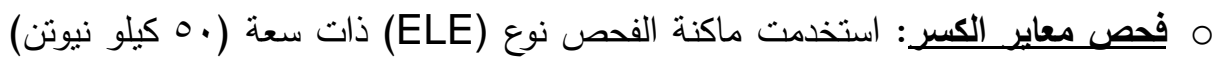
حيث اتبع أسلوب نقطتي التحميل لفحص مقاومة الانثناء وبمسافة امتداد ( ( . ع)ملم،

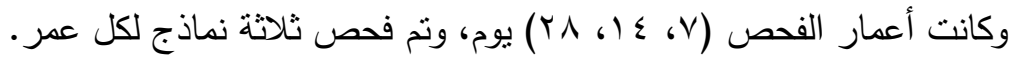

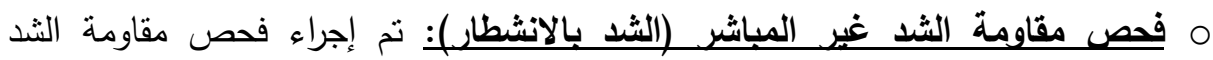

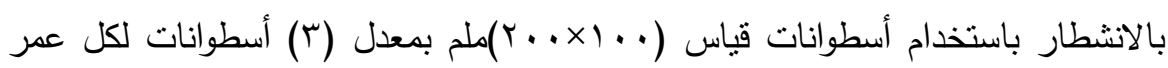
وتم احتساب مقاومة الثد بالانشطار حسب المعادلة التالية:

$\mathrm{Fst}=2 \mathrm{P} / \mathrm{T} \mathrm{dL}$

$$
\begin{aligned}
& \text { مقاومة الثد بالانشطار (MPa) : fst } \\
& \text { (N) : : : : : : : }
\end{aligned}
$$

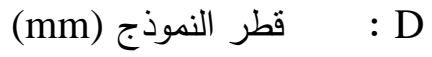

$$
\begin{aligned}
& \text { طول النموذج (mm) : }
\end{aligned}
$$

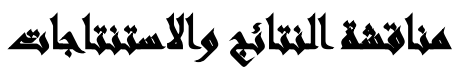

\section{- مقاومة الخرسانة للضغط Concrete Compressive Strength: يوضح}

الجدول رقم (Y) وكل من الأثكال (Y) و (Y) أن هناك تأثنيراً إيجابياً لزيادة نسبة

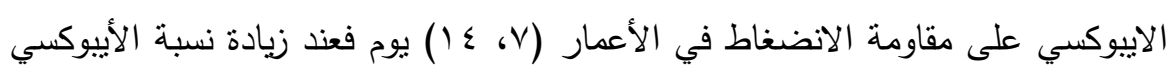

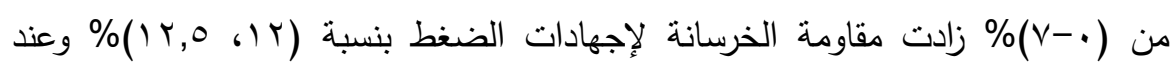




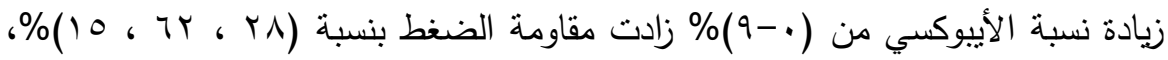

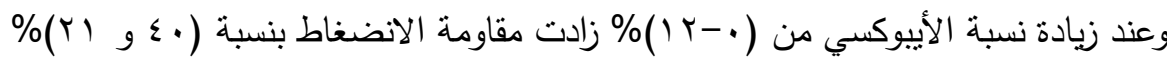

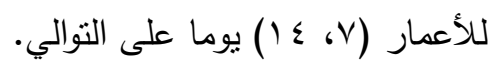

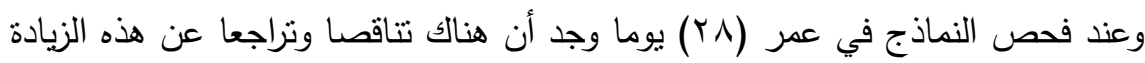

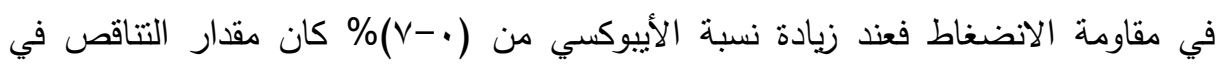

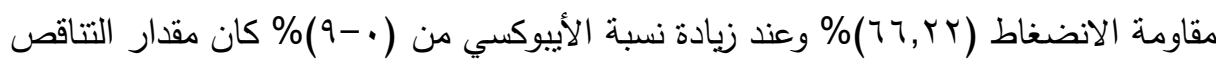

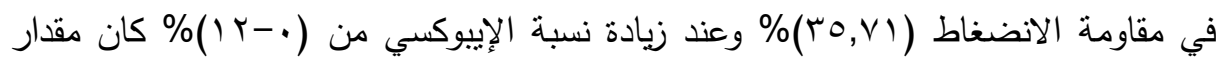

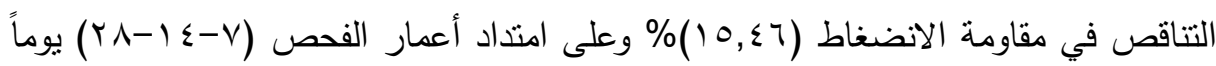

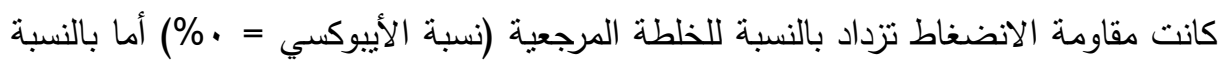

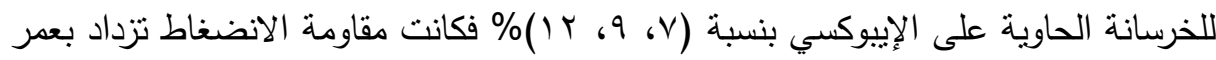

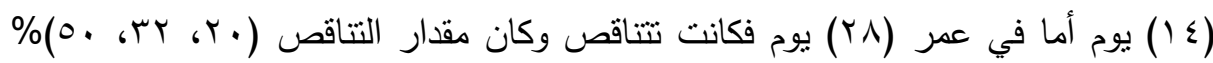

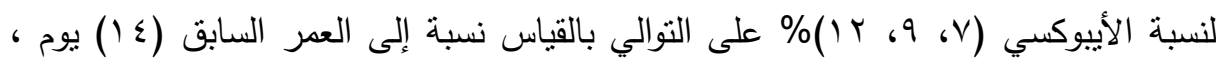

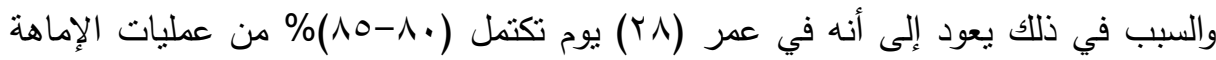
فيتفاعل الاييوكسي مع المكونات الخرسانية مخلفا فجوات أو مسامات تؤدي إلى تقليل كثافة الخلطة الخرسانية فتقل المقاومة. جدول(ץ): معدل مقاومة الانضغاط لنماذج الخلطات المستعطة

\begin{tabular}{|c|c|c|c|c|c|c|c|}
\hline \multicolumn{3}{|c|}{ مقاومة الانضغاط (ميكا } & \multicolumn{4}{|c|}{ نسبة الخلط (الأسمنت، الرمل، الحصى، } & \multirow[t]{2}{*}{ اسم الخلطة } \\
\hline YA & 18 & $V$ & الايبيوكسي & الحصى & الرمل & الأسمنت & \\
\hline$r$. & ד & YA & V & $\Lambda, 0$ & $\Lambda, 0$ & 1 & A \\
\hline rA & rv & Tr & 9 & $\Lambda, 0$ & $\Lambda, 0$ & 1 & B \\
\hline rq & rq & ro & Ir & $\Lambda, 0$ & $\Lambda, 0$ & 1 & C \\
\hline rᄉ & $r T$ & ro & . & 1,0 & $\Lambda, 0$ & 1 & D \\
\hline
\end{tabular}


أحمد خيري حافظ وآخرون
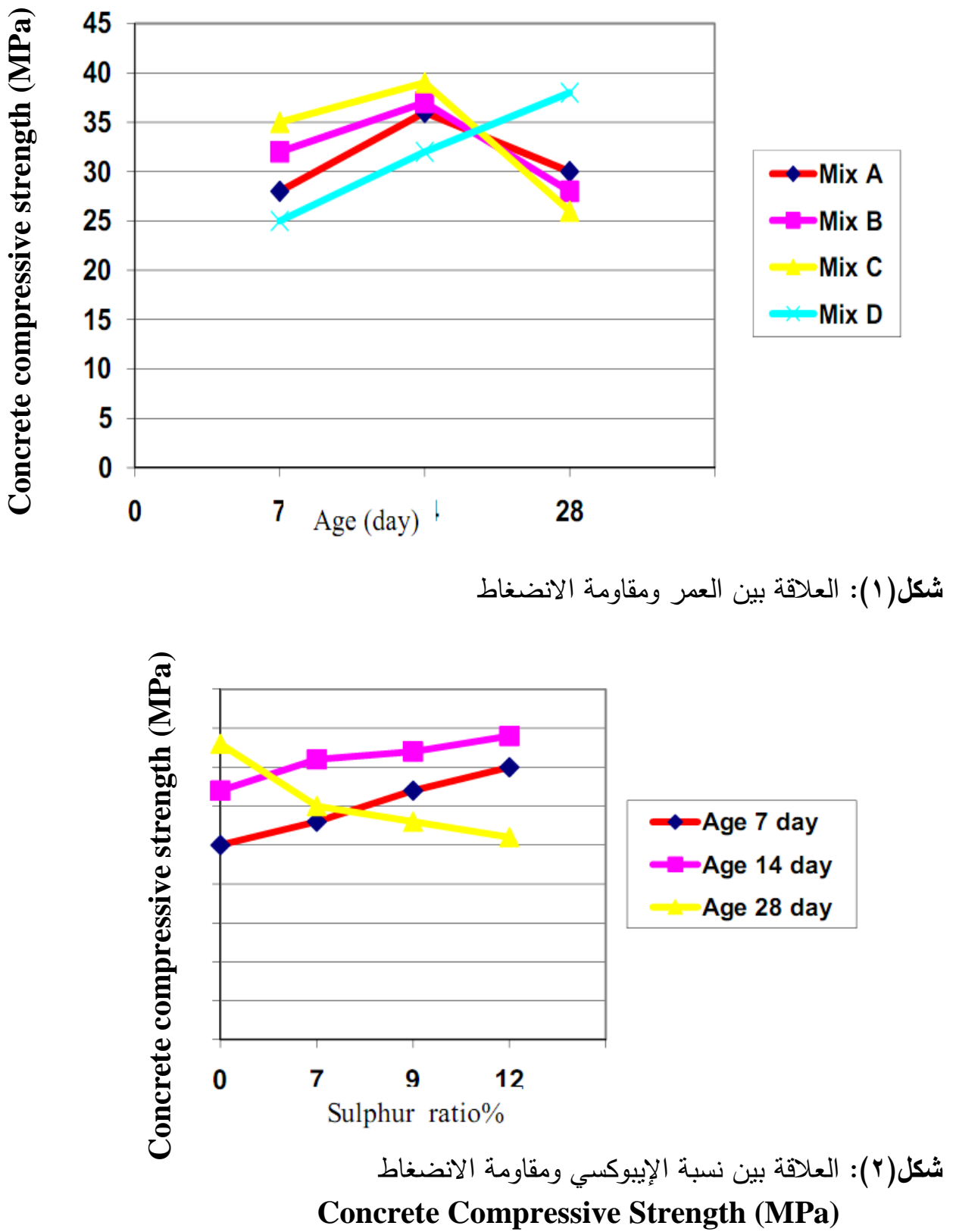

المجلد التاسع والثلاثثن، الجزء الثاني، ديسمبر V V r T 
مقاومة الانحناء Flexural Strength: يبين الجدول (ץ) والأشكال (ץ) و (ع) نتائج فحص مقاومة الاتحناء (معاير الكسر) لكافة الخلطات الخرسانية المستخدمة في هذا البحث حيث نتين هذه النتائج أن مقاومة الانثناء بزيادة العمر للخلطة المرجعية (D) أما للخلطات

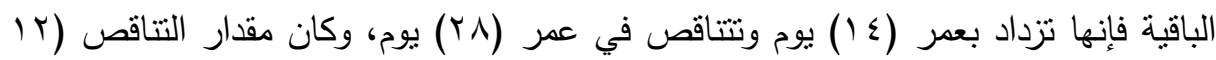

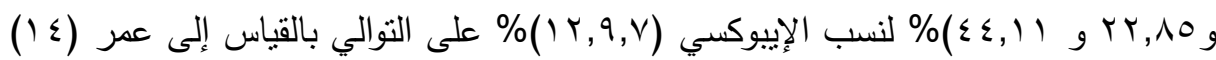
يوم، بينما هناك تأثثر إيجابي لزيادة نسبة الإيبوكسي على مقاومة الإنثاء في الأعمار

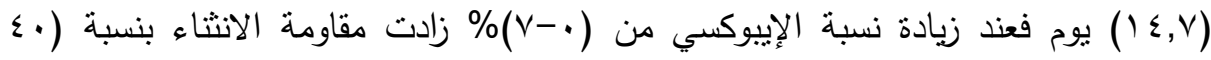

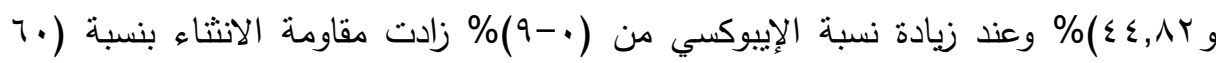

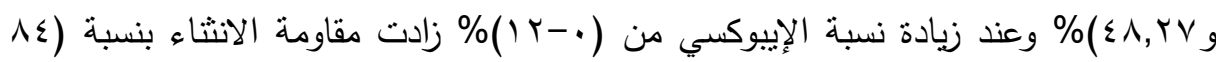

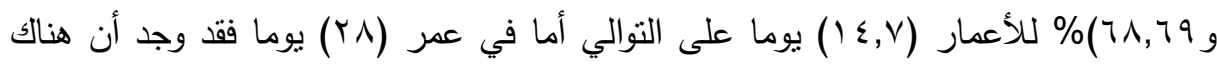

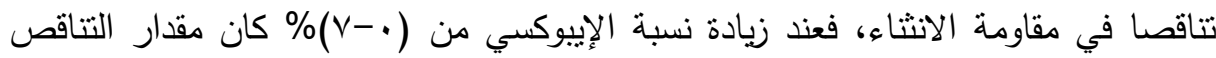

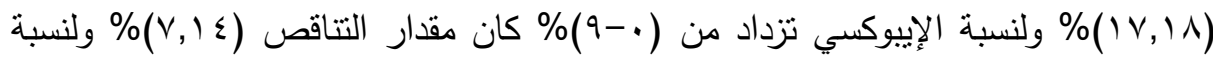

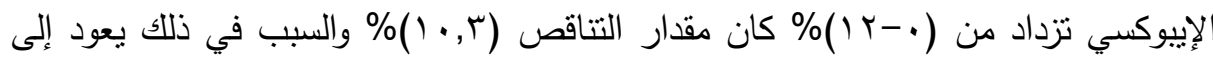

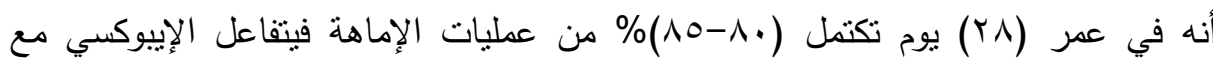
المكونات الخرسانية مخلفا فجوات أو مسامات تؤدي إلى تقليل كثافة الخلطة الخرسانية فتقل

جدول(ץ): معدل مقاومة الانحناء لنماذج الخلطات المستعملة

\begin{tabular}{|c|c|c|c|c|c|c|c|}
\hline \multicolumn{3}{|c|}{ مقاومالة الانضغ حساط (ميكا } & \multicolumn{4}{|c|}{ نسبةٌ الخلط (الأسمنت، الرمل، الحصى، } & \multirow[t]{2}{*}{ اسم الخلطة } \\
\hline$r \Lambda$ & $1 \varepsilon$ & $\mathrm{V}$ & الايبيوكسي & الحصى & الرمل & الأسمنت & \\
\hline $7, \xi$ & $\overline{\Lambda, \xi}$ & $\bar{V}$ & $\bar{V}$ & $\Lambda, 0$ & $\Lambda, 0$ & 1 & $\bar{A}$ \\
\hline $\bar{V}$ & $\Lambda, 7$ & $\Lambda$ & $\bar{q}$ & $\lambda, 0$ & $\Lambda, 0$ & 1 & B \\
\hline $7, \wedge$ & 9,1 & $9, r$ & IY & $\lambda, 0$ & $\Lambda, 0$ & 1 & $\bar{C}$ \\
\hline$V, 0$ & 0,1 & 0 & . & $\Lambda, 0$ & $\Lambda, 0$ & 1 & D \\
\hline
\end{tabular}




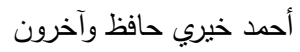

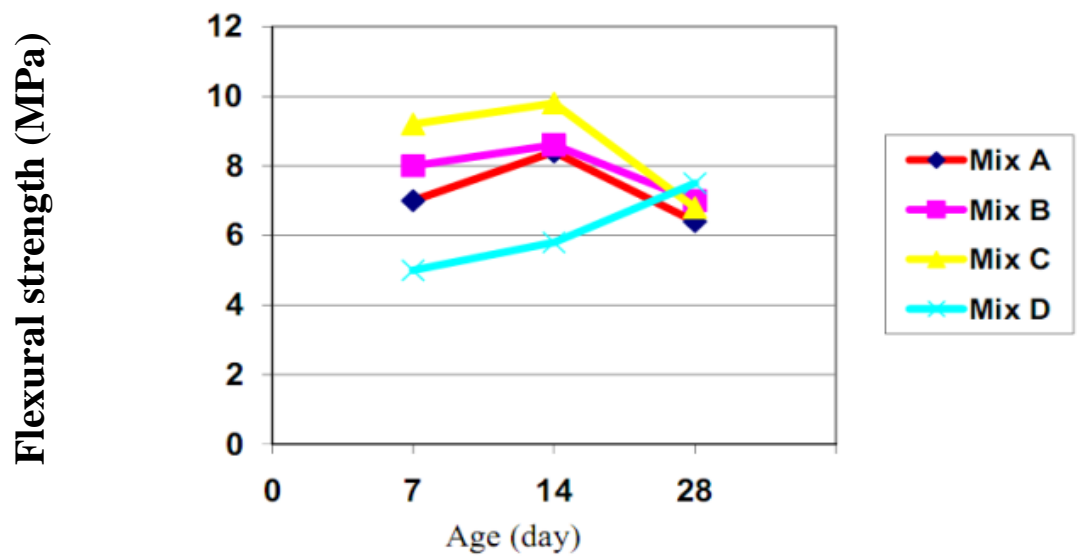

شكل(r): العلاقة بين العمر ومقاومة الانثاء)Flexural Strength (MPa)

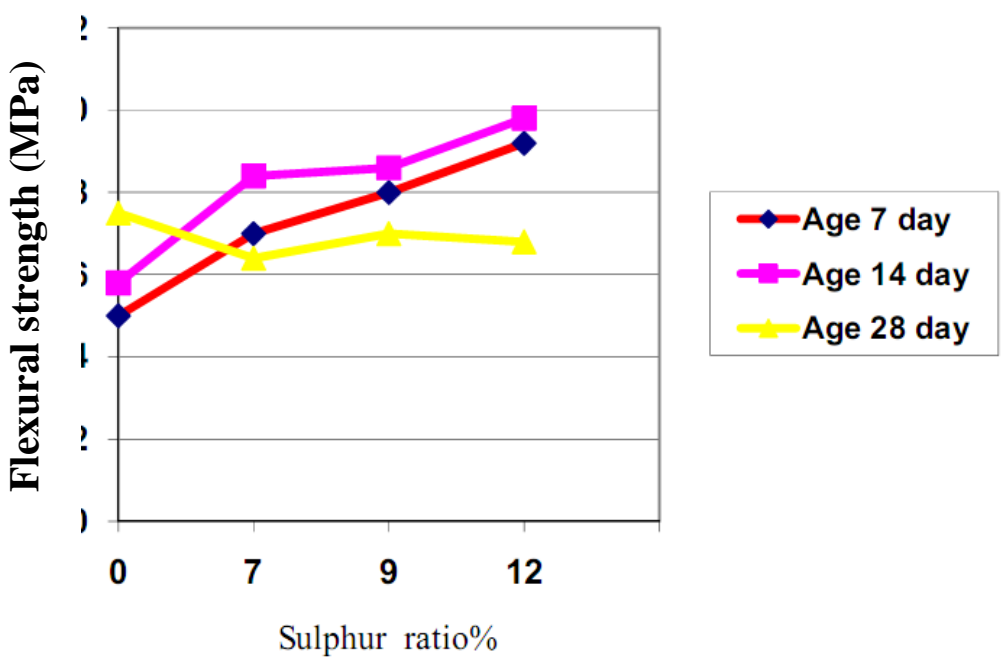

شكل( ) ): العلاقة بين نسبة الإيبوكسي ومقاومة الانثاء Flexural Strength (MPa)

يبين الجدول (ع) نتائج فحوصات الثد الغير مباشر لنماذج هذا البحث فيما نوضح الأثكال (0) و (7) العلاقات التي تمثل هذه النتائج، ويتضح من نلإنك النتائج أن مقاومة الثند غير المباشر تزداد لكافة الخلطات بزيادة عمر الفحص باستثاء الخلطات الحاوية على لده

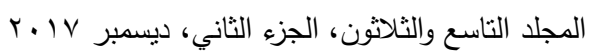


الإييوكسي حيث نتتاقص المقاومة بعمر (Y^) يوم عما عليه في عمر (ع () يوم لهذا النوع

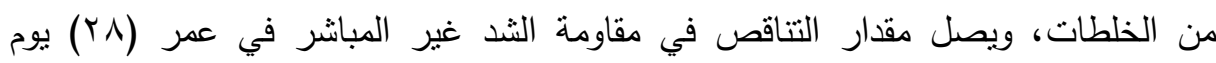

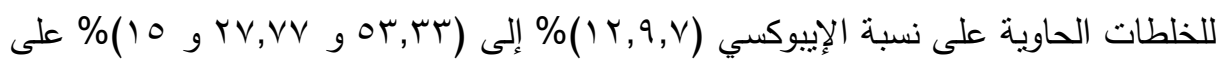

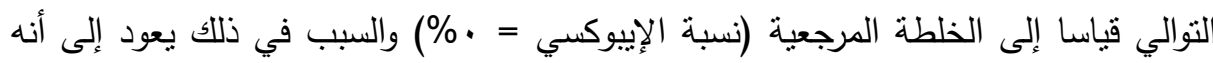

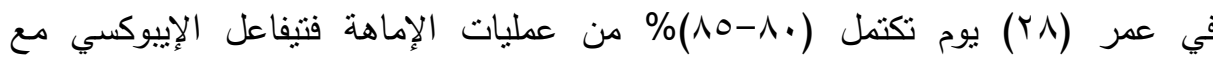
المكونات الخرسانية مخلفا فجوات أو مسامات تؤدي إلى تقليل كثافة الخلطة الخرسانية فتقل

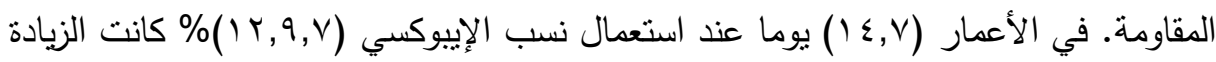

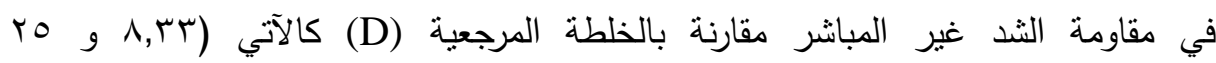

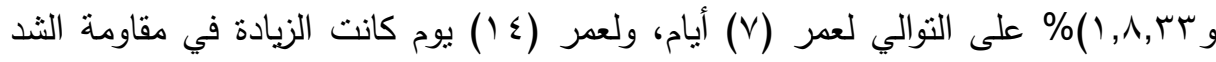

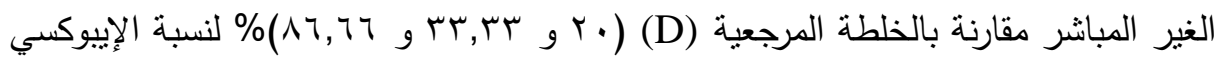
$\%(1 r, q, \vee)$ جدول(§): معدل مقاومة الثد غير المباشر لنماذج الخلطات المستعملة

\begin{tabular}{|c|c|c|c|c|c|c|c|}
\hline \multicolumn{3}{|c|}{ مقاومة الثدا الغير مباشر العمر } & \multicolumn{4}{|c|}{ نسبة الخلط (الأسمنت، الرمل، الحصى، } & \multirow{2}{*}{ 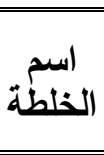 } \\
\hline$r \wedge$ & $1 \varepsilon$ & $\mathrm{V}$ & الاييوكسى & الحصى & الرمل & الأسمنت & \\
\hline 1,0 & $1, \Lambda$ & $1, \Gamma$ & $V$ & $\Lambda, 0$ & $\Lambda, 0$ & 1 & A \\
\hline $1, \wedge$ & r & 1,0 & 9 & $\Lambda, 0$ & 1,0 & 1 & B \\
\hline$r$ & $r, \Lambda$ & $r, 0$ & $1 \%$ & $\Lambda, 0$ & 1,0 & 1 & $\mathrm{C}$ \\
\hline$r, r$ & 1,0 & $1, r$ & & 1.0 & 1.0 & 1 & $\mathrm{D}$ \\
\hline
\end{tabular}


أحمد خيري حافظ وآخرون
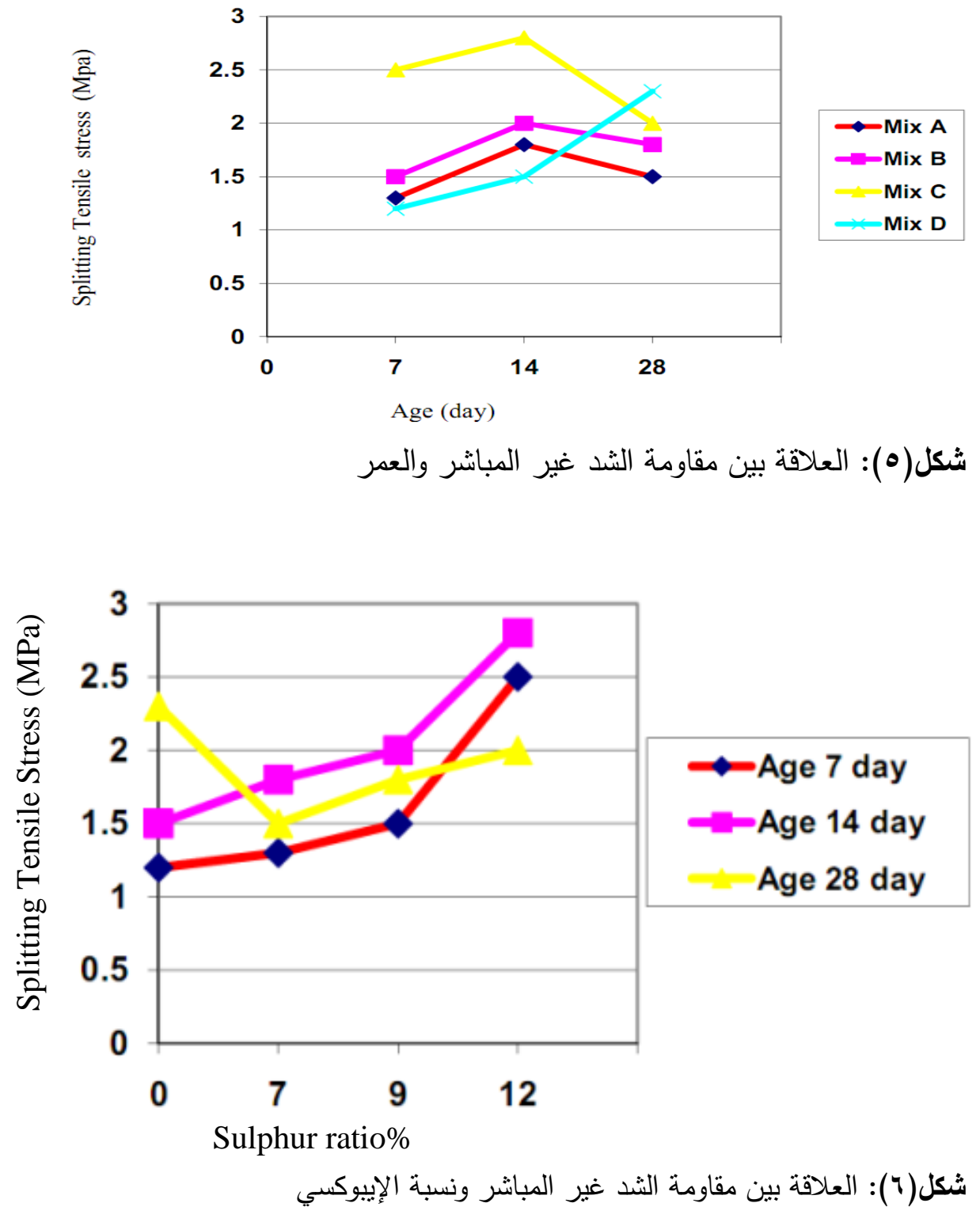

المجلد التاسع والثلاثون، الجزء الثاني، ديسمبر V V r T 


\section{الاستيمتلجايت}

يمكن تلخيص أهم الاسنتتاجات من هذا البحث فيما يلي:

1-زيادة نسبة الإيبوكسي المضاف كبودر ناعم إلى الخلطة الخرسانية يؤدي إلى زيادة مقاومة

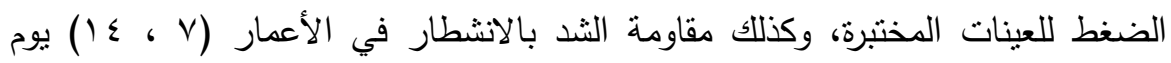

بالخلطة المرجعية غير الحاوية على الإيبوكسي، وتعزى هذه الزيادة إلى أن الإيبوكسي

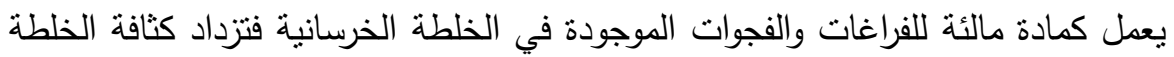

$$
\text { مما يؤدي إلى زيادة المقاومة. }
$$

r-في عمر (r^) يوم تتتاقص مقاومة الضغط، ومقاومة الثد بالانشطار قياسا إلى الخلطة

المرجعية غير الحاوية على الإييوكسي، والسبب في ذلك، يعود إلى: أنه في عمر (Y^)

يوم تكتمل (•^-10)\% من عمليات الإماهة فيتفاعل الإيبوكسي مع المكونات الخرسانية

ويحد من الفجوات والمسامات التي تؤثر على كثافة الخلطة الخرسانية ويزيد من مقاومتها. وفي ضوء نتائج الدراسة، توصي الدراسة بالتالي:

\section{اللزوسيايت}

يمكن استخدام الإيبوكسي لإنتاج الخرسانة الخاصة بنجاح في مجال الإنثاءات مع إضافة مادة مالئة إلى الخلطة الخرسانية لتفادي مشكلة حدوث الفجوات أو المسامات.

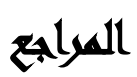

American Society for Testing and Materials, "ASTM Standard test Method for Tensile Properties of Polymer Matrix composite materials, ASTM International, west Conshocken, United states, pp. 1-13, http:// file. yizimg. com/ 175706 / 2012061422194947. pdf (10/10/2017) 
أحمد خيري حافظ وآخرون

Bayan S. Al-Nu'man \& Mohammed Hashim Al-Sahlani., (2006): Behavior of Repaired Reinforced Concrete Beams Failed in Shear, Journal of Engineering and Development, (10), (3), pp. 101-119.

Chung, H. W.,(1975): Three Tests on Epoxy-Repaired Reinforced Concrete Beams, Department of Civil Engineering, University of Hong Kong, China, pp. 205-219.

French, C. W., \& Thorp, G. A., \& Tsai, W. J., (1990): Epoxy repair techniques for moderate earthquake damage, ACI Structural Journal, (87), (4), pp. 416-424 .

Improve (2017): How Much Does An Epoxy Floor Cost, http://www.improvenet.com/r/costs-and-prices/epoxy (14/9/2017)

Jadhav, A. M.,; H, K. Munot.(2016): Analytical Study of Mechanism of Concrete Cracking and Its Propagation Due to Corrosion of Reinforcement in RCC, Open Journal of Civil Engineering, 6: pp. 286-294.

Patil, A.N. (2011): Residual Flexural Strength of RC Beams Subjected to Corrosion. M.Tech Thesis, College of Engineering Pune, pp. 335-342.

Rao, Ganga. H. V. S., \& Taly. N., \& Vijay, P. V.,(2007): Reinforced Concrete Design with FRP Compsite, 1st ed. Taylor \& Francis, pp. 1-335.

Scales, G. M., (1968): Epoxy Resins, ACI Publication (Sp-21).

Zhang, Jinlong.; Bixia, Mai. Tingwei, Cai.; Jiayi, Luo.; Wanhan, Wu.; Bing, Liu.; Ningxu, Han.; Feng, Xing.; Xu Deng (2017) : Optimization of a Binary Concrete Crack Self-Healing System Containing Bacteria and Oxygen, Materials Journal, (10), (116), pp. 1-19. www.mdpi.com/journal/materials $(23 / 4 / 2017)$

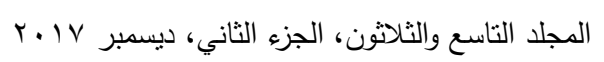




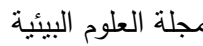

معهد الدراسات والبحوث البيئية - جامعة عين شمس لمس لمس

\title{
EFFECT OF EPOXY IN REDUCING CONCRETE
}

\section{CRACKS}

\author{
Alrifaie, M. M. S. ${ }^{(1)}$ and Ali, Y. R. M. H. ${ }^{(1)}$
}

1) The Public Authority for applied education and training

\begin{abstract}
The study aims to identity the effect of epoxy compound in reducing concrete cracks.

Results of The study:

1- Increasing the percentage of epoxy added as fine powder to the concrete mix, increasing compressive strength, and resistance to tensile at ages (14.7) days, as compared to the mixture that does not contain epoxy, this result is due to the epoxy acts as a filler for the gaps and gaps in the concrete mix, the density of the mixture increases, which lead to increased resistance.

2- At the age of (28) days compression resistance decreasing and tensile resistance to fission compared to the non-epoxy referential mix, the reason for this, back to: that at the age of (28) days completed (80-85)\% dumbness process, epoxy reaction with concrete components, leaving gaps reduce the density of the concrete mix so the resistance is reduced.

The paper recommended that epoxy can be used produce of special concrete in the field of construction with the addition of a filler to the concrete mix to avoid cracks.
\end{abstract}

Key words: Epoxy- Concrete compressive strength - Concrete Cracks concrete tensile strength. 